\title{
Are the Paris' law parameters dependent on each other?
}

\author{
Alberto Carpinteri, Marco Paggi \\ Politecnico di Torino, Dipartimento di Ingegneria Strutturale e Geotecnica, Corso Duca degli Abruzzi 24, \\ 10129 Torino, Italy
}

RIASSUNTO. Nel presente articolo si riesamina la questione relativa all'esistenza di una correlazione tra i parametri $C$ ed $m$ della legge di Paris. In base all'analisi dimensionale ed ai concetti di autosomiglianza incompleta applicati alla fase lineare della propagazione della frattura per fatica, si propone una rappresentazione asintotica che mette in relazione il parametro $C$ ad $m$ ed alle altre variabili che governano il fenomeno in oggetto. Gli esponenti della correlazione vengono poi determinati in base alla condizione che l'instabilità alla Griffith-Irwin debba coincidere con l'instabilità alla Paris nel punto di transizione tra la propagazione sub-critica e quella critica. Si riscontra infine un ottimo accordo tra la correlazione proposta e l'evidenza sperimentale relativamente alle leghe di alluminio, titanio ed acciaio.

ABSTRACT. The question about the existence of a correlation between the parameters $C$ and $m$ of the Paris' law is re-examined in this paper. According to dimensional analysis and incomplete self-similarity concepts applied to the linear range of fatigue crack growth, a power-law asymptotic representation relating the parameter $C$ to $m$ and to the governing variables of the fatigue phenomenon is derived. Then, from the observation that the Griffith-Irwin instability must coincide with the Paris' instability at the onset of rapid crack growth, the exponents entering this correlation are determined. A fair good agreement is found between the proposed correlation and the experimental data concerning Aluminium, Titanium and steel alloys.

KEYWORDS. Fatigue crack growth, Paris' law parameters, Correlation, Dimensional analysis, GriffithIrwin instability.

\section{INTRODUCTION}

Fatigue crack growth data for ductile materials are usually presented in terms of the crack growth rate, $\mathrm{d} a / \mathrm{d} N$, and the stress-intensity factor range, $\Delta K=\left(K_{\max }-K_{\min }\right)$.

At present, it is a common practice to describe the process of fatigue crack growth by a logarithmic $\mathrm{d} a / \mathrm{d} N$ vs. $\Delta K$ diagram (see e.g. Fig. 1).

Three regions are generally recognized on this diagram for a wide collection of experimental results [1]. The first region corresponds to stress-intensity factor ranges near a lower threshold value, $\Delta K_{\text {th }}$, below which no crack propagation takes place. This region of the diagram is usually referred to as Region I, or the near-threshold region [2]. The second linear portion of the diagram defines a power-law relationship between the crack growth rate and the stress-intensity factor range and is usually referred to as Region II [3]. Finally, when $K_{\max }$ tends to the critical stress-intensity factor, $K_{\mathrm{IC}}$, rapid crack propagation takes place and crack growth instability occurs (Region III) [4]. In Region II the Paris' equation $[5,6]$ provides a good approximation to the majority of experimental data:

$$
\frac{\mathrm{d} a}{\mathrm{~d} N}=C(\Delta K)^{m}
$$

where $C$ and $m$ are empirical constants usually referred to as Paris' law parameters.
From the early 60 's, research studies have been focused on the nature of the Paris' law parameters, demonstrating that $C$ and $m$ cannot be considered as material constants. In fact, they depend on the testing conditions, such as the loading ratio $R=\sigma_{\min } / \sigma_{\max }=K_{\min } / K_{\max }$ [7], on the geometry and size of the specimen $[8,9]$ and, as pointed out very recently, on the initial crack length [10]. However, an important question regarding the Paris' law parameters still remains to be answered: are $C$ and $m$ independent of each other or is it possible to find a correlation between them based on theoretical considerations? Concerning this point, it is important to take note of the controversy in the literature about the existence of a correlation between $C$ and $m$. For instance, Cortie [11] stated that the correlation is formal with a little physical relevance, and the high coefficient of correlation between $C$ and $m$ is due to the logarithmic data representation. Similar arguments were proposed in [12], where a correlation-free representation was presented. On the other hand, a very consistent empirical relationship between the Paris' law parameters was found by several Authors [13, 14] and supported by experimental results [3, 13, 15-18]. In this paper, the correlation existing between the Paris' law parameters is derived on the basis of theoretical arguments. To this aim, both self-similarity concepts [9] and the condition that the Paris' law instability corresponds to the Griffith-Irwin instability at the onset of rapid crack growth are profitably used. Comparing the functional expressions derived according to these two in- 


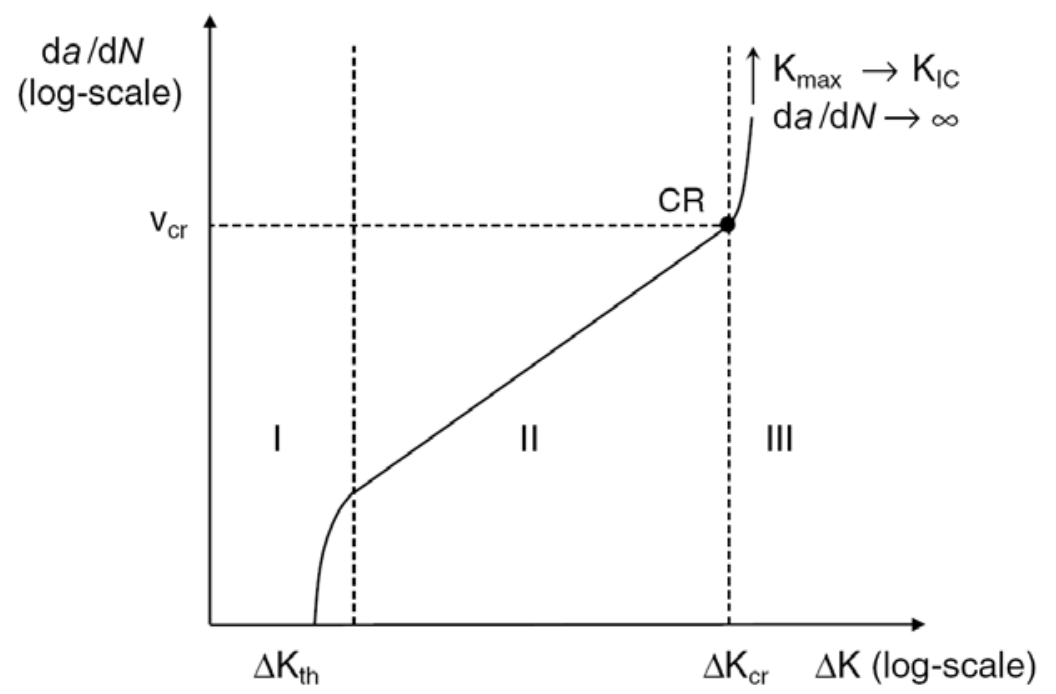

Figure 1. Scheme of the typical fatigue crack propagation curve

\begin{tabular}{|c|l|c|c|}
\hline Variable & \multicolumn{1}{|c|}{ Definition } & Symbol & Dimensions \\
\hline$q_{1}$ & Tensile yield stress of the material & $\sigma_{y}$ & $\mathrm{FL}^{-2}$ \\
$q_{2}$ & Material fracture toughness & $K_{I C}$ & $\mathrm{FL}^{-3 / 2}$ \\
$q_{3}$ & Frequency of the loading cycle & $\omega$ & $\mathrm{T}^{-1}$ \\
\hline$s_{1}$ & Stress-intensity range & $\Delta K=K_{\max }-K_{\min }$ & $\mathrm{FL}^{-3 / 2}$ \\
$s_{2}$ & Characteristic structural size & $D$ & $\mathrm{~L}$ \\
$s_{3}$ & Characteristic internal length & $h$ & $\mathrm{~L}$ \\
$s_{4}$ & Initial crack length & $a_{0}$ & $\mathrm{~L}$ \\
\hline & & $R=\frac{K_{\min }}{K_{\max }}$ & - \\
\hline$r_{1}$ & Loading ratio & & \\
\hline
\end{tabular}

Table 1. Main variables governing the fatigue crack growth phenomenon.

dependent approaches, a relation between the Paris' law parameters $C$ and $m$ is proposed. As a result, it is shown that only one macroscopic parameter is needed for the characterization of damage during fatigue crack growth.

\section{CORRELATION DERIVED ACCORDING TO SELF-SIMILARITY CONCEPTS}

According to dimensional analysis, the physical phenomenon under observation can be regarded as a black box connecting the external variables (called input or governing parameters) with the mechanical response (output parameters). In case of fatigue crack growth in Region II, we assume that the mechanical response of the system is fully represented by the crack growth rate, $q_{0}=\mathrm{d} a / \mathrm{d} N$, which is the parameter to be determined. This output parameter is a function of a number of variables:

$$
q_{0}=F\left(q_{1}, q_{2}, \ldots, q_{n} ; s_{1}, s_{2}, \ldots, s_{m} ; r_{1}, r_{2}, \ldots, r_{k}\right) \text {, }
$$

where $q_{i}$ are quantities with independent physical dimensions, i.e. none of these quantities has a dimension that can be represented in terms of a product of powers of the dimensions of the remaining quantities. Parameters $s_{i}$ are such that their dimensions can be expressed as products of powers of the dimensions of the parameters $q_{i}$. Finally, parameters $r_{i}$ are nondimensional quantities.

As regards the phenomenon of fatigue crack growth, it is possible to consider the following functional dependence:

$$
\frac{\mathrm{d} a}{\mathrm{~d} N}=F\left(\sigma_{y}, K_{\mathrm{IC}}, \varpi ; \Delta K, D, h, a_{0} ; 1-R\right),
$$

where the governing variables are summarized in Tab. 1, along with their physical dimensions expressed in the Length-Force-Time class (LFT). From this list it is possible to distinguish between three main categories of parameters. The first category regards the material parameters, such as the yield stress, $\sigma_{y}$, and the fracture toughness, $K_{\mathrm{IC}}$. The second category comprises the variables governing the testing conditions, such as the stressintensity factor range, $\Delta K$, the loading ratio, $R$, and the frequency of the loading cycle, $\omega$. Concerning environmental conditions and chemical phenomena, they are not considered as primary variables in this formulation and 
their influence on fatigue crack growth can be taken into account as a degradation of the material properties. Finally, the last category includes geometric parameters related to the material microstructure, such as the internal characteristic length, $h$, and to the tested geometry, such as the characteristic structural size, $D$, and the initial crack length, $a_{0}$.

Considering a state with no explicit time dependence, it is possible to apply the Buckingham's $\Pi$ Theorem [19] to reduce by $n$ the number of parameters involved in the problem (see e.g. [8, 20-26] for some relevant applications of this method in Solid Mechanics). As a result, we have:

$$
\begin{aligned}
& \frac{\mathrm{d} a}{\mathrm{~d} N} /\left(\frac{K_{\mathrm{IC}}}{\sigma_{\mathrm{y}}}\right)^{2}= \\
& =\Phi\left(\frac{\Delta K}{K_{\mathrm{IC}}}, \frac{\sigma_{y}{ }^{2}}{K_{\mathrm{IC}}{ }^{2}} D, \frac{\sigma_{y}{ }^{2}}{K_{\mathrm{IC}}{ }^{2}} h, \frac{\sigma_{y}{ }^{2}}{K_{\mathrm{IC}}{ }^{2}} a_{0} ; 1-R\right)= \\
& =\Phi\left(\Pi_{1}, \Pi_{2}, \Pi_{3}, \Pi_{4}, \Pi_{5}\right) .
\end{aligned}
$$

At this point, we want to see if the number of the quantities involved in the relationship (4) can be reduced further from five. Considering the nondimensional parameter $\Delta K / K_{\mathrm{IC}}$, it has to be noticed that this is usually small in the Region II of fatigue crack growth. However, since it is well-known that the fatigue crack growth phenomenon is strongly dependent on this variable (see e.g. the Paris' law in Eq. (1)), a complete self-similarity in this parameter cannot be accepted. Hence, assuming an incomplete self-similarity in $\Pi_{1}$, we have:

$$
\frac{\mathrm{d} a}{\mathrm{~d} N}=\left(\frac{K_{\mathrm{IC}}}{\sigma_{\mathrm{y}}}\right)^{2}\left(\frac{\Delta K}{K_{\mathrm{IC}}}\right)^{\beta_{1}} \Phi_{1}\left(\Pi_{2}, \Pi_{3}, \Pi_{4}, \Pi_{5}\right),
$$

where the exponent $\beta_{1}$ and, consequently, the nondimensional parameter $\Phi_{1}$, cannot be determined from considerations of dimensional analysis alone. Moreover, the exponent $\beta_{1}$ may depend on the nondimensional parameters $\Pi_{i}$. It has to be noticed that $\Pi_{2}$ takes into account the effect of the specimen size and it corresponds to the square of the nondimensional number $Z$ defined in [8], and to the inverse of the square of the brittleness number $s$ introduced in $[20,21,27]$. Moreover, the parameter $\Pi_{4}$ is responsible for the dependence of the fatigue phenomenon on the initial crack length, as recently pointed out in [10].

Repeating this reasoning for the parameter $(1-R)$, which is a small number comprised between zero and unity, a complete self-similarity in $\Pi_{5}$ would imply that fatigue crack growth is independent of the loading ratio. However, this behavior is in contrast with some experimental results indicating an increase in the response $\mathrm{d} a / \mathrm{d} N$ when increasing the parameter $R$ [28]. Therefore, assuming again an incomplete self-similarity in $\Pi_{5}$, we have:

$$
\begin{aligned}
& \frac{\mathrm{d} a}{\mathrm{~d} N}= \\
& =\left(\frac{K_{\mathrm{IC}}}{\sigma_{\mathrm{y}}}\right)^{2}\left(\frac{\Delta K}{K_{\mathrm{IC}}}\right)^{\beta_{1}}(1-R)^{\beta_{2}} \Phi_{2}\left(\Pi_{2}, \Pi_{3}, \Pi_{4}\right)= \\
& =\left(K_{\mathrm{IC}}{ }^{2-\beta_{1}} \sigma_{\mathrm{y}}{ }^{-2}\right)(1-R)^{\beta_{2}} \Delta K^{\beta_{1}} \Phi_{2}\left(\Pi_{2}, \Pi_{3}, \Pi_{4}\right) .
\end{aligned}
$$

Comparing Eq. (6) with the expression of the Paris' law, we find that our proposed formulation encompasses Eq. (1) as a limit case when:

$$
\begin{aligned}
& m=\beta_{1}, \\
& C=\left(K_{\mathrm{IC}}{ }^{2-m} \sigma_{\mathrm{y}}{ }^{-2}\right)(1-R)^{\beta_{2}} \Phi_{2}\left(\Pi_{2}, \Pi_{3}, \Pi_{4}\right) .
\end{aligned}
$$

As a consequence, from Eq. (7) it is possible to notice that the parameter $C$ is dependent on two material parameters, such as the fracture toughness, $K_{\mathrm{IC}}$, and the yield stress, $\sigma_{y}$, as well as on the loading ratio, $R$, and on the nondimensional parameters $\Pi_{2}, \Pi_{3}$, and $\Pi_{4}$. Moreover, Eq. (7) demonstrates, from the theoretical standpoint, the existence of a relationship between the parameters $C$ and $m$.

\section{CORRELATION DERIVED ACCORDING TO THE CRACK GROWTH INSTABILITY CONDITION}

In this Section we derive a correlation between the Paris' law parameters similar to that in Eq. (7) on the basis of the condition of crack growth instability. In fact, as firstly pointed out by Forman et al. [4], the crack propagation rate, $\mathrm{d} a / \mathrm{d} N$, is not only a function of the stress-intensity factor range, $\Delta K$, but also on the condition of instability of the crack growth when the maximum stress-intensity factor approaches its critical value for the material.

Focusing our attention on this dependence, Forman et al. [4] observed that the crack propagation rate must tend to infinity when $K_{\max } \rightarrow K_{\mathrm{IC}}$, i.e.

$$
\lim _{K_{\max } \rightarrow K_{\mathrm{IC}}} \frac{\mathrm{d} a}{\mathrm{~d} N}=\infty
$$

This rapid increase in the crack propagation rate is then responsible for the fast deviation from the linear part of the Region II in the fatigue plot (see e.g. Fig. 1). Considering the transition point labeled CR in Fig. 1 between Region II and Region III, the following relationship between the crack growth rate and the stress-intensity factor range can be derived according to the Paris' law:

$$
\left(\frac{\mathrm{d} a}{\mathrm{~d} N}\right)_{\mathrm{CR}}=v_{\mathrm{CR}}=C\left(\Delta K_{\mathrm{CR}}\right)^{m}
$$


where $\Delta K_{\mathrm{CR}}$ denotes the value of the stress-intensity factor range at the point $C R$. Due to the fact that a rapid variation in the crack propagation rate takes place when the onset of crack instability is reached, it is a reasonable assumption to consider $K_{\max }^{\mathrm{CR}} \cong K_{\mathrm{IC}}$. As a consequence, it is possible to correlate the value of $\Delta K_{\mathrm{CR}}$ with the material fracture toughness:

$$
\Delta K_{\mathrm{CR}}=(1-R) K_{\mathrm{IC}}
$$

Hence, introducing Eq. (10) into Eq. (9), an approximate relationship between the Paris' constants is derived according to the condition that the onset of the Paris' instability corresponds to the Griffith-Irwin instability:

$C \cong v_{\mathrm{CR}}\left[\frac{1}{(1-R) K_{\mathrm{IC}}}\right]^{m}$

Moreover, as regards the parameters $v_{\mathrm{CR}}$ and $K_{\mathrm{IC}}$ entering Eq. (11), it has to be remarked that they are almost constant for each class of material. The dependence on the loading ratio is also put into evidence in Eq. (11).

A closer comparison between Eq. (11) and Eq. (7) permits to clarify the role played by $v_{\mathrm{CR}}$. In fact, Eq. (11) corresponds to the correlation derived according to selfsimilarity concepts when:

$$
\begin{aligned}
m & =\beta_{1}=-\beta_{2}, \\
v_{\mathrm{CR}} & =\left(\frac{K_{\mathrm{IC}}}{\sigma_{\mathrm{y}}}\right)^{2} \Phi_{2}\left(\Pi_{2}, \Pi_{3}, \Pi_{4}\right),
\end{aligned}
$$

confirming the experimental observation reported in [3] that $v_{\mathrm{CR}}$ should depend on the material properties, on the geometry of the tested specimen, and on the material microstructure. Therefore, considering the same testing conditions, this conventional crack growth rate is almost constant for each class of material and Eq. (11) establishes a one-to-one correspondence between the $C$ and $m$ values.

\section{EXPERIMENTAL ASSESSMENT OF THE PROPOSED CORRELATION: ALUMINIUM, TITANIUM AND STEEL ALLOYS}

Parameters $C$ and $m$ entering the Paris' law are usually impossible to be estimated according to theoretical considerations and fatigue tests have to be performed. However, many Authors [3, 13, 29] experimentally observed a very stable relationship between the parameters $C$ and $m$, which is usually represented by the following empirical formula:

$$
C=A B^{m}
$$

usually written in a logarithmic form:

$$
\log C=\log A+m \log B
$$

Taking the logarithm of both sides of the theoretically based relationship between $C$ and $m$ in Eq. (11), we obtain

$$
\log C=\log v_{\mathrm{CR}}+m \log \left[\frac{1}{(1-R) K_{\mathrm{IC}}}\right]
$$

which corresponds to Eq. (14) if

$$
\begin{aligned}
& A=v_{\mathrm{CR}}, \\
& B=\frac{1}{(1-R) K_{\mathrm{IC}}} .
\end{aligned}
$$

In order to check the validity of the proposed correlation derived according to the instability condition of the crack growth, an experimental assessment is performed by comparing the experimentally determined values of $B$ with those theoretically predicted according to Eq. (16).

Concerning steels and Aluminium alloys, Radhakrishnan [13] collected a number of data from various sources and proposed the following least square fit relationships ( $\Delta K$ being in $\mathrm{MPa} \sqrt{\mathrm{m}}$ and $\mathrm{d} a / \mathrm{d} N$ in $\mathrm{m} /$ cycle):

$$
\begin{array}{r}
\log C=\log \left(7.6 \times 10^{-7}\right)+m \log \left(1.81 \times 10^{-2}\right) \\
\text { for steels, } \\
\log C=\log \left(2.5 \times 10^{-6}\right)+m \log \left(4.26 \times 10^{-2}\right) \\
\text { for Al alloys. }
\end{array}
$$

In order to compare the prediction of our proposed correlation with the experimentally determined values of $B$, parameters $m$ and $K_{I C}$ have to be known in advance. However, only in a few studies both the values of the fatigue parameters and of the fracture toughness are experimentally determined and reported. Therefore, to avoid experimental tests, the values of the material fracture toughness are taken from selected handbooks.

Concerning steels, we assume $A=v_{\mathrm{CR}}=7.6 \times 10^{-7}$ $\mathrm{m} / \mathrm{cycle}$, as experimentally determined by Radhakrishnan, $R=0$, and we try to estimate the parameter $B$ on the basis of the values of the fracture toughness proposed in the ASM handbook [30]. This book provides a collection of values in a diagram $K_{I C}$ vs. both the prior austenite grain size, and the temperature test. Over a large range of temperatures ( $T$ from $-269^{\circ} \mathrm{C}$ to $27^{\circ} \mathrm{C}$ ) and grain sizes $(d$ from $1 \mu \mathrm{m}$ to $16 \mu \mathrm{m}), K_{\mathrm{IC}}$ varies from $20 \mathrm{MPa} \sqrt{\mathrm{m}}$ to 100 $\mathrm{MPa} \sqrt{\mathrm{m}}$ with an average value of $K_{\mathrm{IC}}=60 \mathrm{MPa} \sqrt{\mathrm{m}}$. The comparison can also be extended to Aluminium alloys. According to the same procedure discussed above, the estimated average value of the critical stress-intensity factor from handbooks [30-33] is equal to $K_{\mathrm{IC}}=35 \mathrm{MPa} \sqrt{\mathrm{m}}$ with minimum and maximum values equal to $15 \mathrm{MPa} \sqrt{\mathrm{m}}$ and $49 \mathrm{MPa} \sqrt{\mathrm{m}}$, respectively. Using the average values we find:

$$
\log C \cong \log \left(7.6 \times 10^{-7}\right)+m \log \left(1.67 \times 10^{-2}\right)
$$

$$
\text { for steels, }
$$

$$
\log C \cong \log \left(2.5 \times 10^{-6}\right)+m \log \left(2.86 \times 10^{-2}\right)
$$

for $\mathrm{Al}$ alloys. 


\begin{tabular}{|l|r|r|r|c|c|c|}
\hline \multicolumn{1}{|c|}{ Material } & \multicolumn{4}{|c|}{ Experimental data } & \multicolumn{2}{c|}{ Present correlation } \\
\hline & $\begin{array}{c}K_{\mathrm{IC}} \\
(\mathrm{MPa} \sqrt{\mathrm{m}})\end{array}$ & $\begin{array}{c}v_{\mathrm{CR}} \\
(\mathrm{m} / \mathrm{cycle})\end{array}$ & $m$ & $C$ & $C$ & $\begin{array}{c}\text { Relative } \\
\text { error (\%) }\end{array}$ \\
\hline Alum-2219-T62 (L-T) & 28.2 & $3.5 \times 10^{-6}$ & 2.87 & $2.40 \times 10^{-10}$ & $2.41 \times 10^{-10}$ & 0 \\
Alum-2219-T87 (L-T) & 27.3 & $3.5 \times 10^{-6}$ & 3.30 & $6.27 \times 10^{-11}$ & $6.38 \times 10^{-11}$ & 2 \\
Alum-6061-T62 (L-T) & 25.0 & $3.5 \times 10^{-6}$ & 3.20 & $1.63 \times 10^{-10}$ & $1.18 \times 10^{-10}$ & -28 \\
Alum-7075-T73, Forged (L-T) & 27.3 & $3.5 \times 10^{-6}$ & 2.98 & $1.80 \times 10^{-10}$ & $1.84 \times 10^{-10}$ & 2 \\
Pure titanium & 46.0 & $1.0 \times 10^{-5}$ & 3.41 & $1.95 \times 10^{-11}$ & $2.14 \times 10^{-11}$ & 10 \\
(Fty =380 MPa) & 15.5 & $2.0 \times 10^{-7}$ & 3.11 & $3.80 \times 10^{-11}$ & $3.97 \times 10^{-11}$ & 4 \\
Ti-6Al-4V-RT \\
(mill annealed)
\end{tabular}

Table 2. Experimental assessment of the proposed correlation for aluminium, titanium and steel alloys according to the NASGRO database [35].

In both cases, a good agreement between the proposed estimation based on an average value of the critical stress-intensity factor and the experimental relationships in Eq. (17) is achieved.

Another source of experimental data is [34], and is based on the NASGRO program [35], which is one of the most comprehensive database of fatigue crack growth curves for aerospace alloys. These experimental data concern the material fracture toughness, the Paris' law parameters, as well as the crack growth rate corresponding to $K_{\max } \cong K_{I C}$ for fatigue tests characterized by $R=0$ (see Tab. 2).

As previously outlined, the fracture toughness data and the values of $v_{\mathrm{CR}}$ are almost constant for each class of materials. This property is very well evidenced by the 2219-T62, 2219-T87, 6061-T62 and 7075-T73 Aluminium alloys. The application of Eq. (9) permits to predict the value of the Paris' law parameter $C$ as a function of $m$ and to compare it with the experimental one reported in the fifth column of Tab. 2. The agreement between the experimental data and the predictions made according to our correlation is noticeably good, as also evidenced by the relative percentage error reported in the last column of Tab. 2.

\section{CONCLUSIONS}

To shed light on the controversy about the existence of a correlation between the Paris' constants, both selfsimilarity concepts and the condition that the Paris' law instability corresponds to the Griffith-Irwin instability at the onset of rapid crack growth have been profitably used. Comparing the functional expressions derived from these two independent approaches, an approximate relationship between $C$ and $m$ has been proposed. According to this theory, the parameter $C$ is also dependent on the fracture toughness of the material, on the crack growth rate at the onset of crack instability, and on the loading ratio. The main consequence of this correlation is that only one macroscopic parameter is needed for the characterization of damage during fatigue crack growth. A good agreement between the theoretical predictions obtained using this correlations and experimental data has been achieved.

From the engineering standpoint, it has to be emphasized that our proposed correlation constitutes a useful tool for design purposes. In fact, in case of a lack of experimental fatigue data for a new material to characterize, one could, as a first approximation, determine the parameter $C$ as a function of the exponent $m$ according to Eq. (11). Then, a parametric analysis by varying the exponent $m$ in its usual range of variation can be performed and numerical simulations of fatigue crack growth can be put forward. Parameters $v_{\mathrm{CR}}$ and $K_{\mathrm{IC}}$ entering the correlation can be either known in advance, or estimated from materials with similar composition, thermal treatment and mechanical properties (see also [36-38]).

\section{ACKNOWLEDGEMENTS}

Support of the European Union to the Leonardo da Vinci Project "Innovative Learning and Training on Fracture (ILTOF)" is gratefully acknowledged.

\section{REFERENCES}

[1] R.O. Ritchie, "Influence of microstructure on nearthreshold fatigue-crack propagation in ultra-high strength steel”, Metal Science, 11 (1977) 368-381. 
[2] D. Taylor, "Fatigue Thresholds", Butterworths, London (1981).

[3] H. Kitagawa, "Introduction to fracture mechanics of fatigue", In An. Carpinteri, editor, Handbook of fatigue crack propagation in metallic structures, Vol. I, 47-105, Elsevier Science B.V. (1994).

[4] R.G. Forman, V.E. Kearney and R.M. Engle, "Numerical analysis of crack propagation in cyclic-loaded structures", ASME Journal of Basic Engineering, 89 (1967) 459-464.

[5] P.C. Paris, M.P. Gomez and W.P. Anderson, "A rational analytic theory of fatigue", The Trend in Engineering 13 (1961) 9-14.

[6] P.C. Paris and F. Erdogan, "A critical analysis of crack propagation laws", ASME Journal of Basic Engineering, 85D (1963) 528-534.

[7] V.M. Radhakrishnan, "Parameter representation of fatigue crack growth", Engineering Fracture Mechanics, 11 (1979) 359-372.

[8] G.I. Barenblatt and L.R. Botvina, "Incomplete selfsimilarity of fatigue in the linear range of fatigue crack growth" Fatigue and Fracture of Engineering Materials and Structures, 3 (1980) 193-202.

[9] G.I. Barenblatt, "Scaling, self-similarity and intermediate asymptotics", Cambridge University Press, Cambridge (1996).

[10] A. Spagnoli, "Self-similarity and fractals in the Paris range of fatigue crack growth", Mechanics of Materials, 37 (2005) 519-529.

[11] M.B. Cortie, "The irrepressible relationship between the Paris law parameters", Engineering Fracture Mechanics, 30 (1988) 49-58.

[12] F. Bergner and G. Zouhar, "A new approach to the correlation between the coefficient and the exponent in the power law equation of fatigue crack growth", International Journal of Fatigue, 22 (2000) 229-239.

[13] V.M. Radhakrishnan, "Quantifying the parameters in fatigue crack propagation", Engineering Fracture Mechanics, 13 (1980) 129-141.

[14] L. Tóth and A. Krasowsky, "Fracture as the result of self-organised damage process", Journal of Materials Processing Technology, 53 (1995) 441-451.

[15] J.B. Lee and D.N. Lee, "Correlation of two constants in the Paris equation for fatigue crack propagation rate in region II", In Proceedings of the 6th International Conference on Fracture ICF6, New Delhi, India, 1727-1733. Pergamon Press (1984).

[16] M. Cavallini and F. Iacoviello, "Fatigue models for Al alloys", International Journal of Fatigue, 13 (1991) 442-446.

[17] M. Cavallini and F. Iacoviello, "A statistical analysis of fatigue crack growth in a $2091 \mathrm{Al}-\mathrm{Cu}-\mathrm{Li}$ alloy", International Journal of Fatigue, 17 (1995) 135-139.

[18] F. Iacoviello. D. Iacoviello and M. Cavallini, "Analysis of stress ratio effects on fatigue propagation in a sintered duplex steel by experimentation and artificial neural network approaches", International Journal of Fatigue, 26 (2004) 819-828.
[19] E. Buckingham, "Model experiments and the form of empirical equations", ASME Transactions, 37 (1915) 263-296.

[20] A. Carpinteri, "Notch sensitivity in fracture testing of aggregative materials", Engineering Fracture Mechanics, 16 (1982) 467-481.

[21] A. Carpinteri, "Plastic flow collapse vs. separation collapse in elastic-plastic strain-hardening structures", RILEM Materials \& Structures, 16 (1983) 85-96.

[22] A. Carpinteri, "Size effects on strength, toughness and ductility", Journal of Engineering Mechanics, 115 (1989) 1375-1392.

[23] A. Carpinteri, "Cusp catastrophe interpretation of fracture instability", Journal of the Mechanics of Physics of Solids, 37 (1989) 567-582.

[24] A. Carpinteri, "Scaling laws and renormalization groups for strength and toughness of disordered materials", International Journal of Solids and Structures, 31 (1994) 291-302.

[25] A. Carpinteri, "Strength and toughness in disordered materials: complete and incomplete similarity", In SizeScale Effects in the Failure Mechanisms of Materials and Structures, Proceedings of the International Union of Theoretical and Applied Mechanics (IUTAM), Turin, Italy, 3-26, London: E \& FN Spon, (1994).

[26] R.O. Ritchie, "Incomplete self-similarity and fatigue-crack growth", International Journal of Fracture, 132 (2005) 197-203.

[27] A. Carpinteri, "Static and energetic fracture parameters for rocks and concretes", RILEM Materials \& Structures, 14 (1981) 151-162.

[28] I. Milne, R.O. Ritchie and B.L. Karihaloo, eds. "Comprehensive structural integrity: fracture of materials from nano to macro", Vol. 4: Cyclic loading and fatigue, Elsevier, Amsterdam (2003).

[29] E.H. Niccolls, "A correlation for fatigue crack growth rate", Scripta Metallurgica, 10 (1976) 295-298.

[30] Materials Park: ASM International. ASM Handbook, (1985-1988). Fatigue and Fracture.

[31] Materials Park: ASM International. Metals Handbook, (1985).

[32] Materials Park: ASM International 10th Ed. Metals Handbook, (1990). Properties and Selection: Nonferrous Alloys and Special-Purpose Materials.

[33] CINDAS/Purdue University, West Lafayette, IN. Structural Alloys Handbook, (1996).

[34] B. Farahmand, "Analytical method of generating $\mathrm{d} a / \mathrm{d} N$ curves for aerospace alloys", In: Gdoutos EE, editor. Fracture of nano and engineering materials and structures (Proceedings of the 16th European conference of fracture, Alexandroupolis, Greece, 2006), Paper No. 140 on CD-ROM. Springer, Dordrecht (2006).

[35] R.G. Forman, V. Shivakumar and J.C. Newman, "Fatigue crack growth computer program NASA/FLAGRO", Technical Report JSC-22267A, NASA, January 1993.

[36] A. Carpinteri and M. Paggi, "Self-similarity and crack growth instability in the correlation between the 
Paris' constants", Engineering Fracture Mechanics, 74 (2007) 1041-1053.

[37] N. Pugno, M. Ciavarella, P. Cornetti and A. Carpinteri, "A generalized Paris' law for fatigue crack growth", Journal of the Mechanics and Physics of Solids, 54
(2006) 1333-49.

[38] N. Pugno, P. Cornetti and A. Carpinteri, "New unified laws in fatigue: from the Wöhler to the Paris' regime", Engineering Fracture Mechanics, 74 (2007) 595601. 